\title{
Collisional Scaling of the Energytransfer in Drift-Wave Zonal Flow Turbulence
}

\author{
B. Schmid, ${ }^{1, *}$ P. Manz, ${ }^{2,3}$ M. Ramisch, ${ }^{1}$ and U. Stroth ${ }^{2,3}$ \\ ${ }^{1}$ IGVP, Universität Stuttgart, 70569 Stuttgart, Germany \\ ${ }^{2}$ Max-Planck-Institut für Plasmaphysik, 85748 Garching, Germany \\ ${ }^{3}$ Physik-Department E28, Technische Universität München, 85747 Garching, Germany
}

(Dated: July 5, 2016)

\begin{abstract}
The collisionality scaling of density and potential coupling together with zonal flow energy transfer and spectral power is investigated at the stellarator experiment TJ-K. With a poloidal probe array, consisting of 128 Langmuir-probes, density and potential fluctuations are measured on four neighboring flux surfaces simultaneously over the complete poloidal circumference. By analyzing Reynolds stress and pseudo-Reynolds stress it is found, that for increasing collisionality the coupling between density and potential decreases which hinders the zonal flow drive. As a consequence also the nonlinear energy transfer as well as the zonal flow contribution to the complete turbulent spectrum decreases the same way. This is in line with theoretical expectations and is a first experimental verification of the importance of collisionality for large-scale structure formation in magnetically confined toroidal plasmas.
\end{abstract}

PACS numbers: 52.25.Xz, 52.35.Ra, 52.30.-q, 52.55.Hc

Drift waves in toroidal plasmas as well as atmospheric Rossby waves tend to self-generate large scale turbulent structures e.g. zonal flows and jet streams respectively [1-3]. With a poloidal wavenumber $k_{\theta}$ and toroidal wavenumber $k_{\varphi}$ equal zero, and a finite radial extend (i.e. $k_{r} \neq 0$ ), the zonal flow represents an extreme case of a convective cell. Thereby zonal flows are pure potential modes and have no analogous density structure. Like in a self-organization process, Reynolds stresses of the drift waves drive the shear flow, which leads to a self-amplification of the zonal flow [4-6]. In contrast to the rotating fluid system the zonal flow drive in a plasma crucially depends on the cross coupling of potential and density fields. The key parameter in this drift wave zonal flow system is the collisionality $C$ [7]. In the two dimensional Hasegawa-Wakatani equations [8, 9], as a model for drift-wave turbulence, it determines the coupling strength between density and potential, and therefore the efficiency of the driving mechanism. For the adiabatic case $(C \rightarrow 0)$ the two equations reduce to the Hasegawa-Mima equation [10] (Charney equation for Rossby waves [11]), while in the hydrodynamic case $(C \rightarrow \infty)$ density and potential decouple and the zonal flow growth is broken. In this work for the first time, by gradually changing the collisionality, a continuous transition between the two limiting cases has been achieved experimentally and the scaling of the spectral energy transfer into the different channels is studied.

For this investigation a multitude of measurements at different collisionalities have been performed at the low temperature experiment stellarator TJ-K [12]. We find that for lower collisionalities, meaning a more adiabatic electron response, the nonlinear energy transfer into the zonal flow and also the relative zonal flow power in the spectrum strongly increases. This is the first direct experimental measurement of the effect of collisionality $C$ on the zonal flow drive.

As a characteristic of zonal flows is the homogeneous potential perturbation on a complete flux surface, a poloidal probe array was used to reliably distinguish zonal flows from the residual turbulence. The array consists of 128 Langmuir-probes with 32 probes on each of four neighboring magnetic flux surfaces and is shown in Fig. 1 (a) as used in [13]. It is designed for an outer port
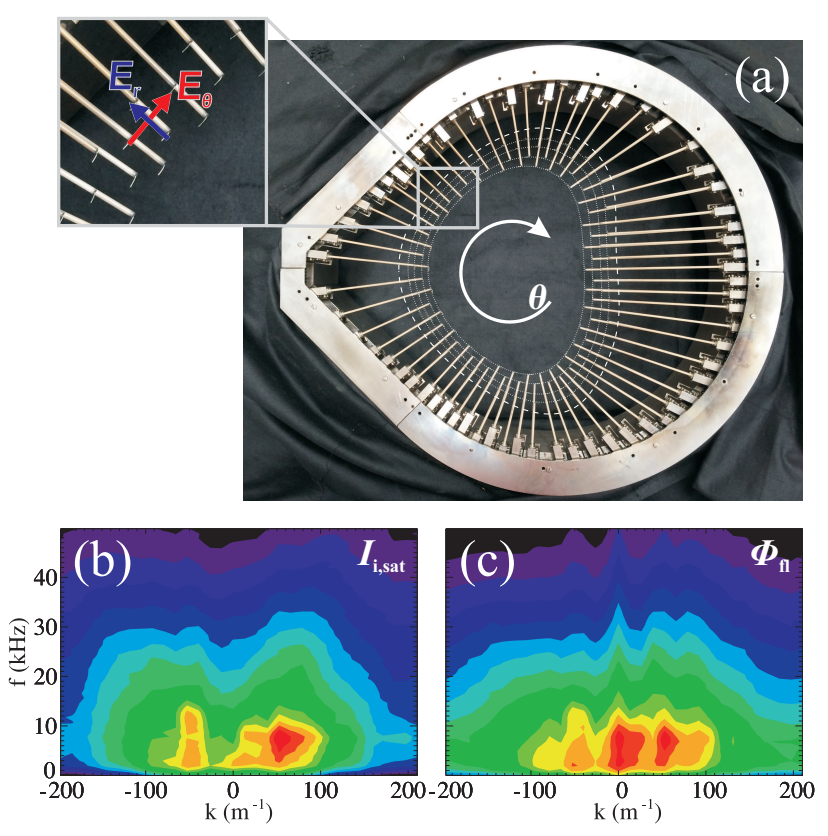

FIG. 1. (color online). (a) Picture of the poloidal probe array with Langmuir probes on four flux surfaces. The inlet illustrates the measurement of radial and poloidal electric field with which the Reynolds stress can be calculated. Logarithmic wavenumber frequency spectra of density (b) and potential (c) are shown below. The $k_{\theta}=0$ mode is the zonal flow, which is not present in the density. 
of the stellarator TJ-K with triangular cross section, and placed in the confined region just inside the separatrix (dashed white line), where the gradients are steepest. The probes are spaced nonuniformly in order to get a perpendicular orientation of probes on every other flux surface. The average poloidal probe spacing is $\mathrm{d} x=1.37$, $1.49,1.61$, and $1.73 \mathrm{~cm}$ on the four different flux surfaces at normalized radii $\rho=0.81,0.85,0.89$, and 0.94 with a radial separation of $\mathrm{d} r \approx 0.5 \mathrm{~cm}$. Also with a spatial uncertainty of $2 \mathrm{~mm}$, the distances are still below the typical structure size of 3 to $5 \mathrm{~cm}$ [14-16]. The use of Langmuir probes makes it possible to acquire data with $1 \mathrm{MHz}$ and up to $2^{20}$ samples for all probes simultaneously. For the poloidal probe array it is possible to switch the operation mode for all 128 probes individually from $-90 \mathrm{~V}$ probe bias to a floating probe measuring ion saturation current or floating potential, respectively. Since temperature fluctuations are small in TJ-K [17], fluctuations in the ion saturation current can be associated with density fluctuations $\left(\tilde{I}_{\mathrm{i} \text { sat }} \propto \tilde{n}\right)$ and floating potential fluctuations with plasma potential fluctuations $\left(\tilde{\phi}_{\mathrm{fl}} \approx \tilde{\phi}_{\mathrm{pl}}\right)$ [18]. Using two neighboring probes, the electric field is measured and the poloidal $\mathrm{E} \times \mathrm{B}$ drift velocity is given by $v^{E \times B} \approx\left(\tilde{\phi}_{\mathrm{fl}}^{i+1}-\tilde{\phi}_{\mathrm{fl}}^{i}\right) /(B \mathrm{~d} x)$. Measuring both velocity components in the poloidal cross section, the Reynolds stress is then given as the product of fluctuations in radial $\widetilde{v}_{r}$ and poloidal $\widetilde{v}_{\theta}$ velocity

$$
\mathrm{R}=\left\langle\widetilde{v}_{r} \widetilde{v}_{\theta}\right\rangle \approx\left\langle\frac{\left(\tilde{\phi}_{\mathrm{fl}}^{\theta_{i+1}}-\tilde{\phi}_{\mathrm{fl}}^{\theta_{i}}\right)\left(\tilde{\phi}_{\mathrm{fl}}^{r_{i+1}}-\tilde{\phi}_{\mathrm{fl}}^{r_{i}}\right)}{r \mathrm{~d} \theta \mathrm{d} r B^{2}}\right\rangle .
$$

With the poloidal probe array the flux surface averaged (indicated by $\langle$.$\rangle ) Reynolds stress can be measured$ on two different flux surfaces, giving the possibility to get a direct estimate of the zonal flow drive given by $\partial_{t}\left\langle v_{\theta}\right\rangle=-\partial_{r} \mathrm{R}[19]$.

In the same discharge, using a movable triple probe, radial profiles of the ion saturation current, floating potential and electron temperature $T_{\mathrm{e}}$ are obtained, latter from fitting the probe characteristic. The line-averaged density of the microwave interferometer was used to get absolute density values $n_{e}$. In order to cover a broad collisionality range 90 plasma experiments were performed with ion masses ranging from $m_{i}^{\mathrm{H}} \approx 1 \mathrm{u}$ up to $m_{i}^{\mathrm{Kr}} \approx 84 \mathrm{u}$. At a $2.45 \mathrm{GHz}$ microwave heating frequency and a corresponding magnetic field of $B=72 \mathrm{mT}$ [20], the neutral gas pressure $p_{0}$ and microwave power $P_{\mathrm{MW}}$ were varied resulting in densities between $n_{e} \approx 0.7-3.0 \cdot 10^{17} \mathrm{~m}^{-3}$ and electron temperatures ranging from $T_{e} \approx 3.9$ to $14.4 \mathrm{eV}$. For each discharge the collisionality was calculated according to [9]

$$
C=\frac{\hat{\nu}}{\hat{k}_{\|}^{2}} \propto \frac{B n_{e}}{k_{\|}^{2} m_{i} T_{e}^{5 / 2}},
$$

where $\hat{\nu}$ is the normalized collision frequency and $\hat{k}_{\|}$the normalized parallel wavelength [21]. Mainly by changing
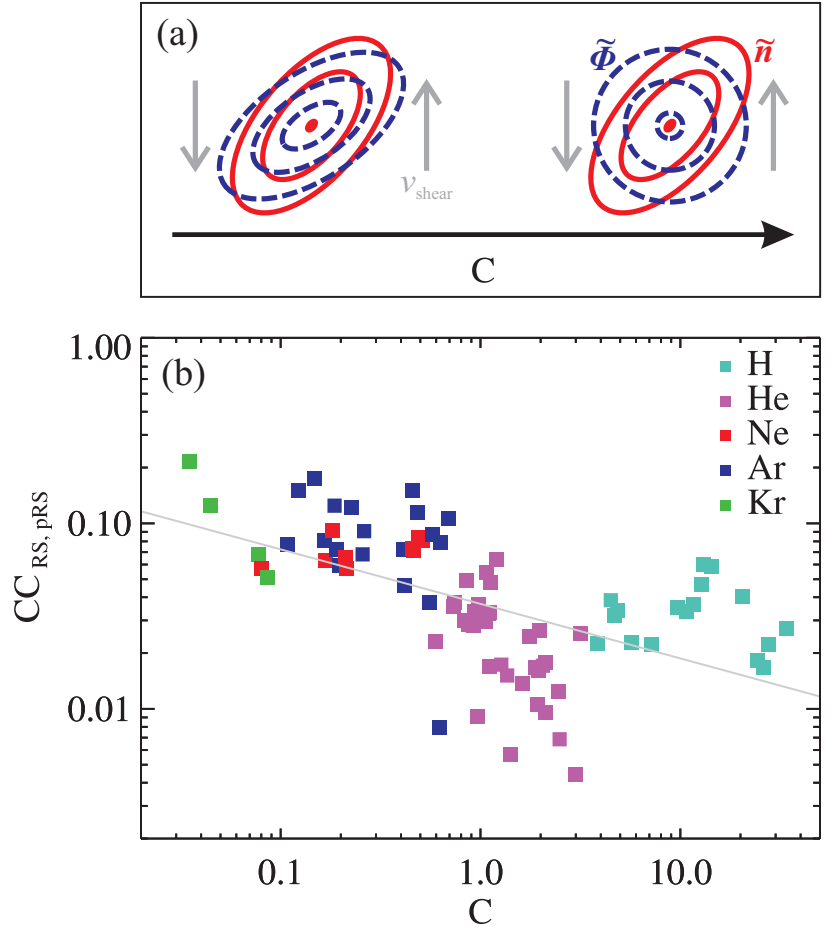

FIG. 2. (color online). The graphic (a) illustrates the change of density (red, solid lines) and potential (dashed, blue lines) coupling with collisionality $C$. For high collisionality density and potential decouple and the tilt is not transferred to the potential anymore. The changed density potential coupling is also shown by the correlation of Reynolds stress and pseudoReynolds stress, which is shown in (b).

ion mass, the collisionality could be varied by about two orders of magnitude, which makes it possible to study the transition from the hydrodynamic regime $(C \gg 1)$ to the adiabatic regime $(C \ll 1)$.

Figure 1 (b) and (c) show typical wavenumber frequency spectra ( $k f$-spectra) of ion saturation current and floating potential measured on the 2nd and 3rd flux surface in a helium discharge. The broad turbulent spectrum is dominated by turbulent modes with positive wavenumbers indicating a propagation into the electron diamagnetic drift direction, which are associated with drift waves. A dominant mode number of $m=4$ is plausible since drift waves have finite parallel wavelength $\left(k_{\|} \neq 0\right)$ and the experiment has a rotational transform of $t \approx 1 / 4[21,22]$. The $k_{\theta}=0$ mode in the potential spectrum is apparent, while not present in the density. This is the signature of the zonal flow, which is known to be a pure potential mode and it also excludes the possibility of a pure mean background fluctuation, since the density is not changed.

The zonal flow driving mechanism is based on the shearing of the drift-wave eddies in a background shear flow [23]. The collisionality is thereby the control parameter which determines the efficiency of this driving mech- 
anism. In a simple model with cold ions the drift-wave turbulence can be described with the two dimensional Hasegawa-Wakatani equations [8, 9],

$$
\begin{aligned}
\partial_{t} n+\{\phi, n\}+\kappa_{n} \partial_{y} \phi & =C^{-1}(\phi-n), \\
\partial_{t} \Omega+\{\phi, \Omega\} & =C^{-1}(\phi-n) .
\end{aligned}
$$

Here $n, \phi$ and $\Omega$ denote the normalized density, the potential and the vorticity fluctuations, respectively. $\kappa_{n}$ is the normalized background density gradient and $\{.,$. represents Poisson brackets. Equations (3) and (4), essentially originating from the electron continuity equation and quasineutrality, respectively, are coupled via the parallel electron dynamics included in the collisionality $C$. For an adiabatic electron response (adiabatic regime $C \ll 1$ ) density and potential act similar, as for the hydrodynamic regime $(C \gg 1)$ the two equations decouple and density and potential act as separate fluids. This dependency is illustrated in Fig. 2 (a) by an eddy in a background shear flow. The spatial shapes of the density (red, solid lines) and the potential perturbation (dashed, blue lines) are shown for low and high collisionality $C$. A background shear flow (e.g. the zonal flow) tilts the vortex in the density, and through parallel coupling also the potential is deformed. In the limit of high collisionality the vortex tilt in the density is not transferred to the potential anymore. In magnetized plasma the potential perturbation leads, via $\mathrm{E} \times \mathrm{B}$ drift, to vortices perpendicular to the magnetic field. A sheared eddy has a non isotropic velocity distribution, giving a nonzero Reynolds stress, and in turn leading to an amplification of the initial shear flow. With this argumentation it is clear, that with an increased collisionality the zonal flow drive is hindered.

To get an estimate for the coupling of density and potential, the so called pseudo-Reynolds stress, originally introduced to get information about the Reynolds stress from density measurements [24], is calculated. The density is thereby treated analog to the potential field. From Eqs. (3) and (1) it then follows, that the density-based pseudo-Reynolds stress has to be corrected by terms of at least linear order in the collisionality $\mathcal{O}(C)$ leading to the following relation between Reynolds stress $R_{\phi}$ and pseudo-Reynolds stress $\mathrm{R}_{n}$,

$$
\begin{aligned}
\mathrm{R}=\mathrm{R}_{\phi} & =\left\langle\frac{\left(\tilde{n}^{\theta_{i+1}}-\tilde{n}^{\theta_{i}}\right)\left(\tilde{n}^{r_{i+1}}-\tilde{n}^{r_{i}}\right)}{r \mathrm{~d} \theta \mathrm{d} r B^{2}}\right\rangle+\mathcal{O}(C) \\
& =\mathrm{R}_{n}+\mathcal{O}(C)
\end{aligned}
$$

With a different bias setting of the poloidal probe array it is possible to measure Reynolds stress and pseudoReynolds stress at the same time over the poloidal circumference with a reduced spatial resolution. Probes measuring ion saturation current alternate with probes on floating potential when going around the circumference. Since Reynolds stress and pseudo-Reynolds stress
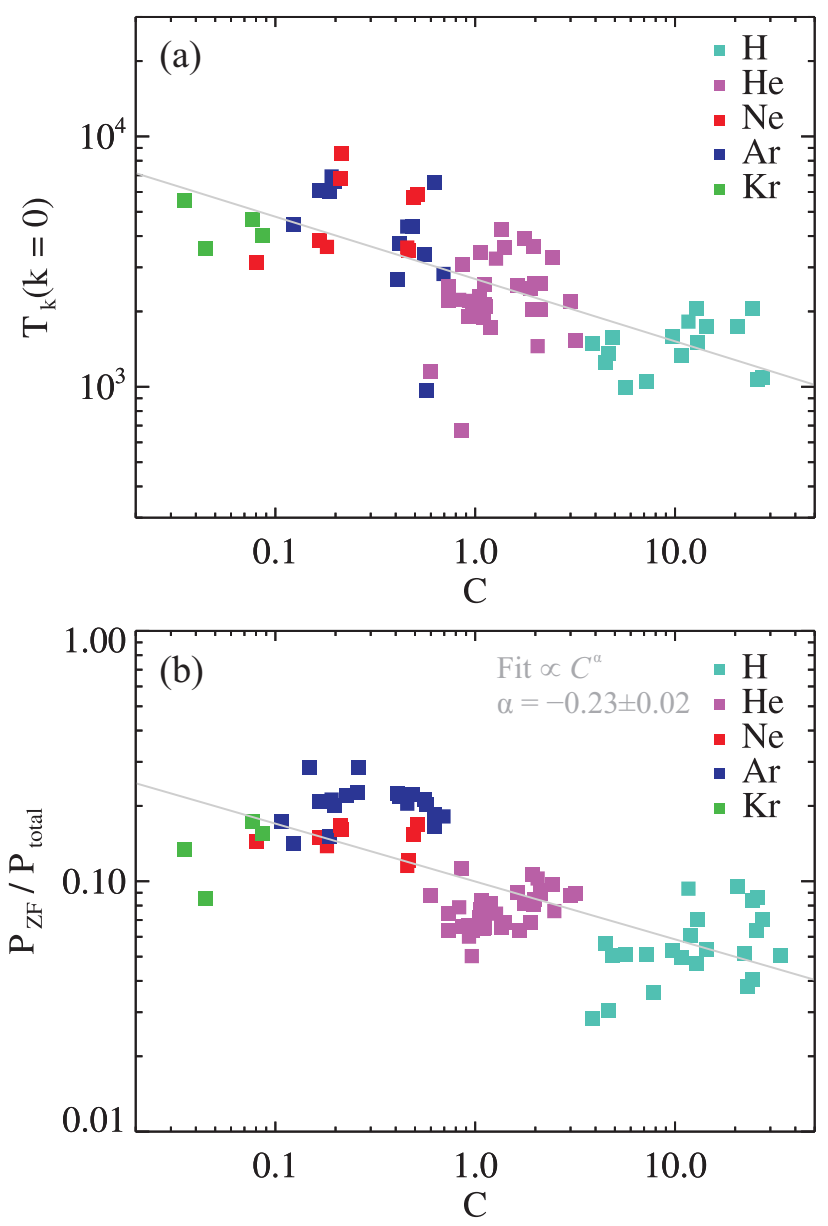

FIG. 3. (color online). The scaling of the spectral transfer of density fluctuation activity with collisionality (a). Relative zonal flow power for the same experiments (b) (see text for further information).

can be measured on two flux surfaces, the corresponding zonal flow drive $-\partial_{r} \mathrm{R}$ of both quantities is obtained. The two time traces of $2^{20}$ samples for each discharge have been cross-correlated and the scaling of the maximal correlation between Reynolds-stress drive and pseudo Reynolds-stress drive with collisionality is shown in Fig. 2 (b). Although the correlation values are small, they are significant and show a clear trend. For lower collisionality the correlation between both parameters increases, pointing to an increased coupling between density and potential.

It was shown that the turbulence in the stellarator TJ-K is drift-wave dominated [21, 25-27] and for the following analysis of the energy transfer it is assumed that the nonlinear wave-coupling equation is satisfied,

$$
\begin{aligned}
\frac{\partial \varphi(k, t)}{\partial t} & =\Lambda_{k}^{L}(k) \varphi(k, t) \\
& +\frac{1}{2} \sum_{k=k_{1}+k_{2}} \Lambda_{k}^{Q}\left(k_{1}, k_{2}\right) \varphi\left(k_{1}, t\right) \varphi\left(k_{2}, t\right) .
\end{aligned}
$$


Here $\varphi(k, t)$ is a fluctuating quantity, $\Lambda_{k}^{L}(k)$ the linear coupling coefficient and $\Lambda_{k}^{Q}\left(k_{1}, k_{2}\right)$ the quadratic coupling coefficients [28]. With a discretization in time $\Delta t$, equation (6) leads to a set of momentum equations which are then solved using the modified Ritz method introduced by Kim et al. [29]. This approach to solve the wave-coupling equation also considers fourth-order moments in order to avoid a closure approximation. For the transfer of spectral power $P_{k}$, the wave kinetic equation can be derived,

$$
\frac{\partial}{\partial t} P_{k}=2 \gamma_{k} P_{k}+\sum_{k=k_{1}+k_{2}} T_{k}\left(k_{1}, k_{2}\right) .
$$

Since the zonal flow is nonlinearly driven by the turbulence, the nonlinear spectral power transfer function $T_{k}\left(k_{1}, k_{2}\right)$ is of interest for the subsequent analysis, which is given as

$$
T_{k}\left(k_{1}, k_{2}\right)=\operatorname{Re}\left(\Lambda_{k}^{Q}\left(k_{1}, k_{2}\right)\left\langle\varphi\left(k_{1}, t\right) \varphi\left(k_{2}, t\right) \varphi^{*}(k, t)\right\rangle_{t}\right) .
$$

It should be stressed, that the quantities used here are directly calculated in wavenumber space and a conditional averaging technique is used to calculate the temporal ensemble average $\langle.\rangle_{t}$. Especially the power transfer in the density fluctuation activity, where the nonlinearity in the $\mathrm{E} \times \mathrm{B}$ drift is included [7], is considered for the scaling. Therefore the fluctuating quantities are assigned to $\varphi\left(k_{1}, t\right)=n\left(k_{1}, t\right), \varphi\left(k_{2}, t\right)=n\left(k_{2}, t\right)$, and $\varphi^{*}(k, t)=\phi(k, t)[30]$. To omit a reduction in $k$-space resolution, the ion saturation current and the floating potential are measured simultaneously on the second and third flux surface, respectively. For the zonal flow $(k=0)$ a positive energy transfer into it is found for all measurements, confirming the inverse energy transfer originally published in [4] obtained by the Camargo method [7]. In Fig. 3 (a) the scaling over the collisionality of this energy transfer is shown for several discharges with a variety of gases. With lower collisionality the energy transfer to the zonal flow increases, showing the increased drive by the drift-wave turbulence. To see if an increased energy transfer into the zonal flow leads to an increased zonal flow activity, the total power of the zonal flow is calculated. As the overall turbulence strongly depends on the background gradients, not the absolute zonal flow power is interesting, but the relative contribution to the turbulent spectrum. Therefore the relative zonal flow power shown in Fig. 3 (b) is calculated as

$$
P_{Z F} / P_{\text {total }}=\sum_{f \leq 8 \mathrm{kHz}} \mathrm{S}_{\phi}(k=0, f) / \sum_{k, f} \mathrm{~S}_{\phi}(k, f)
$$

from the wavenumber frequency spectrum $\mathrm{S}_{\phi}(k, f)$. As indicated above for the zonal flow component only the low frequency bandpass filtered spectral power is used. In the adiabatic limit $(C \rightarrow 0)$, the zonal flow contribution to the complete spectrum strongly increases and the relative power reaches values of up to $29 \%$ of the total turbulent spectral power. To deduce a quantitative assertion, the collisionality scaling of the relative zonal flow power is fitted with a power law $\propto C^{\alpha}$, where we find a value of $\alpha=-0.23 \pm 0.02$.

In summary, the collisionality dependence of the zonal flow drive was studied in great detail. With the possibility of Reynolds stress measurements in real space, it was found, that for increasing collisionality the coupling between density and potential decreases, which in return makes the zonal flow driving mechanism less effective. As a result also the nonlinear energy transfer into the zonal flow, as well as the relative spectral power of the zonal flow decrease with higher collisionality. This is a direct test of fundamental mechanism in plasma turbulence on a microscopic level of plasma turbulent fluctuations and also represents a first verification of the importance of collisionality for large-scale structure formation in magnetically confined toroidal plasmas.

We thank S.-I. Itoh and K. Itoh for fruitful discussions.

* Bernhard.Schmid@igvp.uni-stuttgart.de

[1] A. Hasegawa, C. G. Maclennan, and Y. Kodama, Physics of Fluids 22, 2122 (1979).

[2] J. D. Meiss, Physics of Fluids 26, 990 (1983).

[3] M. Heimpel, J. Aurnou, and J. Wicht, Nature 438, 193 (2005).

[4] P. Manz, M. Ramisch, and U. Stroth, Physical Review Letters 103, 165004 (2009).

[5] P. Manz, G. Birkenmeier, M. Ramisch, and U. Stroth, Physics of Plasmas 19, 082318 (2012).

[6] G. Birkenmeier, M. Ramisch, B. Schmid, and U. Stroth, Physical Review Letters 110, 145004 (2013).

[7] S. J. Camargo, D. Biskamp, and B. D. Scott, Physics of Plasmas 2, 48 (1995).

[8] A. Hasegawa and M. Wakatani, Physical Review Letters 50, 682 (1983).

[9] M. Wakatani and A. Hasegawa, Physics of Fluids 27, 611 (1984).

[10] A. Hasegawa and K. Mima, Physical Review Letters 39, 205 (1977).

[11] J. G. Charney, Geofys. Publ. 17, 3 (1942).

[12] N. Krause, C. Lechte, J. Stöber, U. Stroth, E. Ascasibar, J. Alonso, and S. Niedner, Review of Scientific Instruments 73, 3474 (2002).

[13] P. Manz, M. Ramisch, and U. Stroth, Physics of Plasmas 16, 042309 (2009)

[14] M. Ramisch, N. Mahdizadeh, U. Stroth, F. Greiner, C. Lechte, and K. Rahbarnia, Physics of Plasmas 12, 032504 (2005)

[15] G. Fuchert, G. Birkenmeier, B. Nold, M. Ramisch, and U. Stroth, Plasma Physics and Controlled Fusion 55, 125002 (2013).

[16] G. Birkenmeier, M. Ramisch, G. Fuchert, A. Köhn, B. Nold, and U. Stroth, Plasma Physics and Controlled Fusion 55, 015003 (2013).

[17] S. Enge, G. Birkenmeier, P. Manz, M. Ramisch, and U. Stroth, Physical Review Letters 105, 175004 (2010). 
[18] N. Mahdizadeh, F. Greiner, M. Ramisch, U. Stroth, W. Guttenfelder, C. Lechte, and K. Rahbarnia, Plasma Physics and Controlled Fusion 47, 569 (2005).

[19] P. H. Diamond and Y. Kim, Physics of Fluids B: Plasma Physics 3, 1626 (1991).

[20] A. Köhn, G. Birkenmeier, E. Holzhauer, M. Ramisch, and U. Stroth, Plasma Physics and Controlled Fusion 52, 035003 (2010).

[21] N. Mahdizadeh, F. Greiner, T. Happel, A. Kendl, M. Ramisch, B. D. Scott, and U. Stroth, Plasma Physics and Controlled Fusion 49, 1005 (2007).

[22] M. Ramisch, F. Greiner, N. Mahdizadeh, K. Rahbarnia, and U. Stroth, Plasma Physics and Controlled Fusion 49, 777 (2007).

[23] U. Stroth, P. Manz, and M. Ramisch, Plasma Physics and Controlled Fusion 53, 024006 (2011).

[24] S. B. Korsholm, P. K. Michelsen, V. Naulin, J. J. Ras- mussen, L. Garcia, B. a. Carreras, and V. E. Lynch, Plasma Physics and Controlled Fusion 43, 1377 (2001).

[25] C. Lechte, S. Niedner, and U. Stroth, New Journal of Physics 4, 334 (2002).

[26] U. Stroth, F. Greiner, C. Lechte, N. Mahdizadeh, K. Rahbarnia, and M. Ramisch, Physics of Plasmas 11, 2558 (2004).

[27] K. Rahbarnia, E. Holzhauer, N. Mahdizadeh, M. Ramisch, and U. Stroth, Plasma Physics and Controlled Fusion 50, 085008 (2008).

[28] C. P. Ritz, E. J. Powers, and R. D. Bengtson, Physics of Fluids B: Plasma Physics 1, 153 (1989).

[29] J. S. Kim, R. D. Durst, R. J. Fonck, E. Fernandez, A. Ware, and P. W. Terry, Physics of Plasmas 3, 3998 (1996).

[30] P. Manz, M. Xu, S. C. Thakur, and G. R. Tynan, Plasma Physics and Controlled Fusion 53, 095001 (2011). 